03

\title{
Тензостимулированный эффект в легированном и термообработанном кремнии при ориентированной деформации
}

\author{
(C) О.О. Маматкаримов ${ }^{1}$, О. Химматкулов ${ }^{2}$, И.Г. Турсунов ${ }^{3,4}$ \\ ${ }^{1}$ Наманганский инженерно-технологический институт, \\ Наманган, Узбекистан \\ 2 Ташкентский государственный технический университет, \\ Ташкент, Узбекистан \\ 3 Чирчикский государственный педагогический институт, \\ Чирчик, Узбекистан \\ ${ }^{4}$ Национальный университет Узбекистана, \\ Ташкент, Узбекистан \\ E-mail: ikromjon0804@gmail.com \\ Поступила в Редакцию 17 декабря 2020 г. \\ В окончательной редакции 17 декабря 2020 г. \\ Принята к публикации 14 января 2021 г.
}

\begin{abstract}
Исследован тензостимулированный эффект в термообработанном и легированном кремнии. Показано, что одноосное сжатие по направлению [111] приводит к распаду термодоноров и проявлению гистерезиса в зависимостях удельного сопротивления от величины сжатия. Установлено, что тензостимулированный эффект в компенсированных марганцем образцах кремния обусловлен одновременными изменениями концентрации и подвижности носителей тока.
\end{abstract}

Ключевые слова: деформация, тензосопротивления, кремний, легирование, одноосное.

DOI: 10.21883/FTT.2021.05.50807.262

\section{1. Введение}

Как известно, в сильно компенсированном кремнии легированном примесями, создающими в запрещенной зоне глубокие энергетические уровни, наблюдается ряд уникальных по свойству магнитных, оптических и других явлений $[1,2]$. Сильно компенсированные атомами марганца монокристаллы кремния и структуры на их основе являются чувствительными к внешней упругой деформации и могут быть использованы в качестве чувствительных элементов датчиков внешнего давления $[3,4]$.

Исследованию влияния одноосной упругой деформации на электрофизические свойства в полупроводниках посвящен ряд работ [5-7]. Но в литературе имеется мало сведений об особенностях тензостимулированного эффекта изменения удельного сопротивления при наличии глубоких энергетических уровней в запрещенной зоне и термодефектов в объеме полупроводника. Как известно $[8,9]$ после термического отжига при высоких температурах и резкого охлаждения в объеме полупроводникового кристалла образуются электроактивные и нейтральные термические дефекты. Исследованиями ряда авторов [10-12] установлено, что термоотжиг различной длительности $(1-200 \mathrm{~h})$ в широком интервале температур (300-1200 $\left.{ }^{\circ} \mathrm{C}\right)$ кислородосодержащих монокристаллов кремния, выращенных методом Чохральского, приводит к образованию различных термодоноров (TD) кислородной природы в объеме кристалла $\mathrm{Cz}-\mathrm{Si}$. Образование термодоноров в большинстве случаев при- писывается возникновению комплексов кислорода с атомами кремния, структурными дефектами и примесными атомами. В этом случае TD обнаруживаются по влиянию на концентрацию, время жизни и подвижности носителей тока - по изменению оптических спектров поглощения и микротвердости, а также структурными анализами.

Проведенные спектроскопические исследования методами эффекта Холла, ЭПР, ИК-поглощения, DLTS идр. показали, что TD в отожженных монокристаллах $\mathrm{Si}$ представляют квазинепрерывный набор донорных состояний в запрещенной зоне кремния с уровнями залегания в интервале (10-300 meV) [13]. Высокотемпературные TD связывают с преципитатами кислорода внедрения, углерод в положении замещения $\mathrm{C}_{s}$ выступает в роли центра зарождения крупных преципитатов кислорода. Преципитатам больших размеров соответствуют более глубокие уровни вблизи зоны проводимости. Вопросы определения коэффициента диффузии кислорода в кремнии и переориентации кислородосодержащих двойных термодоноров в условиях одноосного сжатия приведены и в работах $[14,15]$.

В настоящей работе исследовано влияние термообработки и примесных состояний марганца на эффект тензосопротивления в кремнии.

\section{2. Методика эксперимента}

В качестве исходных образцов были использованы бездислокационные монокристаллы кремния марки 
Электрофизические параметры компенсированного кремния

\begin{tabular}{c|c|c|c|c|c|c|c}
\hline № & $\begin{array}{c}\text { Марка } \\
\text { исходного кремния }\end{array}$ & $T,{ }^{\circ} \mathrm{C}$ & $t, \mathrm{~h}$ & Образец & $\rho, \Omega \cdot \mathrm{cm}$ & $\mu, \mathrm{cm}^{2} / \mathrm{V} \cdot \mathrm{s}$ & $p, \mathrm{~cm}^{-3}$ \\
\hline 1 & КДБ-4 & - & - & $p$-Si $\langle\mathrm{B}\rangle$ & 4 & 300 & $5.34 \cdot 10^{15}$ \\
2 & КДБ-4 & 1150 & 2 & $p$-Si $\langle\mathrm{B}, \mathrm{TD}\rangle$ & 40 & 200 & $7.77 \cdot 10^{14}$ \\
3 & КДБ-4 & 1090 & 2 & $p$-Si $\langle\mathrm{B}, \mathrm{Mn}\rangle$ & $7.7 \cdot 10^{2}$ & 250 & $3.25 \cdot 10^{13}$ \\
4 & КДБ-4 & 1135 & 2 & $p$-Si $\langle\mathrm{B}, \mathrm{Mn}\rangle$ & $3.62 \cdot 10^{5}$ & 150 & $1.15 \cdot 10^{11}$
\end{tabular}

КДБ-4, выращенные методом Чохральского, с концентрацией кислорода $(5-6) \cdot 10^{17} \mathrm{~cm}^{-3}$. Компенсированные образцы $\mathrm{Si}\langle\mathrm{B}, \mathrm{Mn}\rangle$ были получены методом высокотемпературной диффузии марганца из газовой фазы. Термообработанные образцы $\mathrm{Si}\langle\mathrm{B}, \mathrm{TD}\rangle$ изготовлялись путем отжига исходных монокристаллов в условиях вакуума с последующим резким охлаждением со скоростью $100-200^{\circ} \mathrm{C} / \mathrm{s}$. Технологические параметры процессов диффузии и термоотжига, а также электрофизические параметры определенные из холловских измерений приготовленных образцов приведены в таблице. Изотермическое одноосное сжатие осуществляли в направлении [111] при температуре $T=300 \mathrm{~K}$.

Проведенные эксперименты показали, что термообработка (ТО) в интервале температур $1000-1200^{\circ} \mathrm{C}$ и последующее охлаждение со скоростью $100-200^{\circ} \mathrm{C} / \mathrm{s}$ приводит к существенному изменению электрических параметров кремния (таблица). После ТО и охлаждения удельное сопротивление исходных образцов $p$-Si $\langle\mathrm{B}\rangle$ с $\rho=4 \Omega \cdot \mathrm{cm}$ изменяется на один порядок. Исходные $p$ - $\mathrm{Si}\langle\mathrm{B}\rangle$, термообработанные $p$ - $\mathrm{Si}\langle\mathrm{B}, \mathrm{TD}\rangle$ и компенсированные $p$-Si $\langle\mathrm{B}, \mathrm{Mn}\rangle$ образцы были подвергнуты направленному сжатию вдоль кристаллографической оси [111] в интервале давлении $X=(0-6) \cdot 10^{8} \mathrm{~Pa}$.

В таблице приведены электрофизические параметры компенсированного кремния.

\section{3. Результаты и их обсуждение}

Экспериментальные исследования показали, что с ростом ориентированного давления удельное сопротивление термообработанных образцов $p$-Si $\langle\mathrm{B}\rangle$ с удельным сопротивлением $\rho=40 \Omega \cdot \mathrm{cm}$ уменьшается, а при снятии сжатия в зависимостях $\rho(X)$ наблюдается гистерезис, т.е. после снятия одноосного давления конечное значение $\rho$ уменьшается по сравнению с его начальным значением (рис. 1 , кривые $1, l^{\prime}$ ).

Полученные экспериментальные результаты показывают, что наблюдаемый гистерезис в зависимостях $\rho(X)$ обусловлен аналогичным поведением подвижности дырок от одноосного сжатия, связанной с распадом крупномасштабных примесных скоплений в виде преципитатов (кластеров) кислорода, образованных при термообработке в объеме кристалла кремния.

В исходных $p$-Si $\langle\mathrm{B}\rangle$ образцах с $\rho=4 \Omega \cdot \mathrm{cm}$ удельное сопротивление монотонно уменьшается с ростом сжатия $X$ (рис. 1, кривая 2). Удельное сопротивление

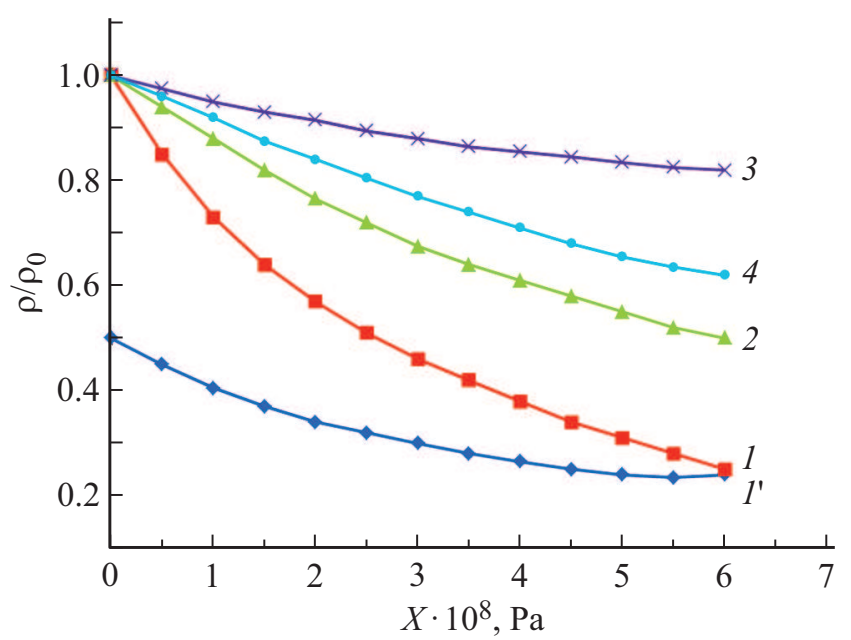

Рис. 1. Тензосопротивление образцов $p$ - $\mathrm{Si}\langle\mathrm{B}\rangle$ при одноосном сжатии при $T=300 \mathrm{~K}: 1-\mathrm{TO} p-\mathrm{Si}\langle\mathrm{B}, \mathrm{TД}\rangle, \rho=40 \Omega \cdot \mathrm{cm}$, $l^{\prime}-\mathrm{TO} p$ - $\mathrm{Si}\langle\mathrm{B}, \mathrm{TД}\rangle, \rho=40 \Omega \cdot \mathrm{cm}$ при снятии одноосного давления, 2 - исходный $p$ - $\mathrm{Si}\langle\mathrm{B}\rangle, \rho=4 \Omega \cdot \mathrm{cm}, 3-p$ - $\mathrm{Si}\langle\mathrm{B}, \mathrm{Mn}\rangle$, $\rho=7.7 \cdot 10^{2} \Omega \cdot \mathrm{cm}, 4-p-\mathrm{Si}\langle\mathrm{B}, \mathrm{Mn}\rangle, \rho=3.62 \cdot 10^{5} \Omega \cdot \mathrm{cm}$.

компенсированных и сильнокомпенсированных (СК) образцов так же уменьшается с ростом сжатия $X$ ( рис. 1 , кривые 3 и 4).

Исследования тензо-Холл-эффекта, проведенные с целью выявления механизма тензосопротивления в данных образцах, показали, что концентрация в исходных и термообработанных образцах во всем диапазоне сжатия остается постоянной (рис. 2, кривые 1 и 2), так как мелкие примесные уровни при комнатной температуре являются полностью опустошенными.

Подвижность носителей тока в исходных $p$-Si $\langle\mathrm{B}\rangle$ образцах с удельными сопротивлениями $\rho=4 \Omega \cdot \mathrm{cm}$ с ростом $X$ монотонно увеличивается (рис. 3, кривая 2), что хорошо согласуется с существующими представлениями теории тензоэффекта [5]. Согласно этим представлениям одноосное сжатие в направлении [111] приводит к расщеплению валентной зоны кремния и перераспределению носителей заряда из зоны тяжелых в зону легких дырок. Как известно, в условиях постоянной температуры подвижность дырок при рассеивании на колебаниях решетки (оптических фононах) и ионах примеси зависит в основном от их эффективной массы как $\sim \mathrm{m}^{-5 / 2}$ и $\sim \mathrm{m}^{-1 / 2}$ соответственно [16]. Отсюда следует, что при изотермическом одноосном сжатии в изменении подвижности дырок основной вклад дает 


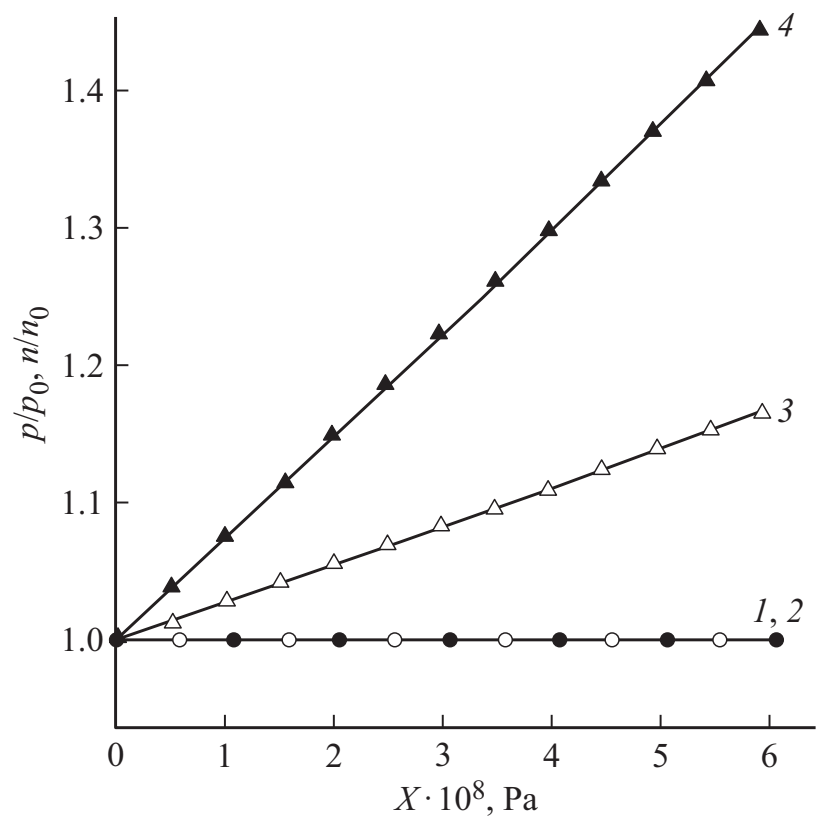

Рис. 2. Зависимости концентрации дырок в образцах $p$-Si $\langle\mathrm{B}\rangle$ от одноосного сжатия при условиях $I\|X\|[111]$ 1 - ТО $p$-Si $\langle\mathrm{B}, \mathrm{TD}\rangle, \rho=40 \Omega \cdot \mathrm{cm}, 2$ - исходный $p$-Si $\langle\mathrm{B}\rangle$, $\rho=4 \Omega \cdot \mathrm{cm}, \quad 3-p-\mathrm{Si}\langle\mathrm{B}, \mathrm{Mn}\rangle, \rho=7.7 \cdot 10^{2} \Omega \cdot \mathrm{cm}, \quad 4-$ $p-\mathrm{Si}\langle\mathrm{B}, \mathrm{Mn}\rangle, \rho=3.62 \cdot 10^{5} \Omega \cdot \mathrm{cm}$.

их рассеивание на оптических фононах. Следовательно, можно предположить, что уменьшение эффективной массы дырок приводит к росту их подвижности с ростом сжатия.

При снятии сжатия в зависимостях $\mu(X)$ дырок в термообработанных образцах $p$-Si $\langle\mathrm{B}\rangle$ с удельным сопротивлением $\rho=4 \Omega \cdot \mathrm{cm}$ также наблюдается гистерезис, и конечное значение подвижности дырок возрастает (рис. 3, кривые $1,1^{\prime}$ ). Такое изменение подвижности в ТО-образцах при одноосном упругом сжатии, хотя по характеру и совпадает с данными теории тензоэффекта, по которому рост подвижности дырок объясняется расщеплением валентной зоны, но столь значительный ее рост и появление гистерезиса в зависимости $\mu(X)$ позволяет предположить, что помимо указанного механизма изменения подвижности при деформации существует дополнительный эффект, сильно влияющий на подвижность.

Как было сказано выше, термообработка при высоких температурах и резкое охлаждение приводит к образованию в объеме монокристаллического кремния неоднородностей, связанных с медленно диффундирующими примесями, т.е. примесных скоплений и микродефектов, которые уменьшают подвижности носителей тока. Уменьшение подвижности дырок наблюдается и в наших образцах, подвергшихся ТО. В связи с этим, изменение подвижности дырок в ТО-образцах при их одноосном сжатии можно связать с проявлением эффекта барического распада нейтральных примесных скоплений и микродефектов, который приводит к увеличению по- движности дырок и гистерезису в зависимостях $\rho(X)$ и $\mu(X)$.

В компенсированных (рис. 2, кривая 3) и СК-образцах (рис. 2, кривая 4) $\mathrm{Si}\langle\mathrm{Mn}\rangle$, концентрация дырок увеличивается с ростом $X$, причем сравнительно заметный рост наблюдается в СК-образцах $\mathrm{Si}\langle\mathrm{Mn}\rangle$. Увеличение концентрации в образцах $\mathrm{Si}\langle\mathrm{Mn}\rangle$ возможно связано с изменением степени заполнения глубоких уровней $\mathrm{Mn}$ в $\mathrm{Si}$ за счет изменения их энергии ионизации при одноосном сжатии [17-19].

Из рис. 3 видно, что значение подвижности дырок в компенсированных образцах $\mathrm{Si}\langle\mathrm{Mn}\rangle$ (кривая 3) увеличивается, а в СК-образцах $\mathrm{Si}\langle\mathrm{Mn}\rangle$ уменьшается с ростом $X$ (кривая 4). Уменьшение подвижности дырок в CK-образцах $\mathrm{Si}\langle\mathrm{Mn}\rangle$ при сжатии вдоль кристаллографической оси [111] не согласуется с теорией тензоэффекта, поэтому предполагается, что наблюдаемое уменьшение подвижности носителей тока является результатом проявления „встречного“ существующему механизму деформации другого механизма, приводящего к уменьшению подвижности [20]. По-видимому, это связано с усилением рассеяния носителей тока за счет увеличения радиуса экранирования электрического потенциала неоднородностей в объеме кристалла Si при деформации в направлении кристаллографической оси [111], так как деформация является анизотропной. В условиях сильной компенсации межузельные примесные атомы марганца полностью ионизованы и в основном находятся в зарядовых состояниях $\mathrm{Mn}^{+}$и $\mathrm{Mn}^{++}$, которые являются мощными отталкивающими центрами для дырок [19]. В сильно компенсированных полупроводниках случайное распределение примесей по кристаллу приводит к

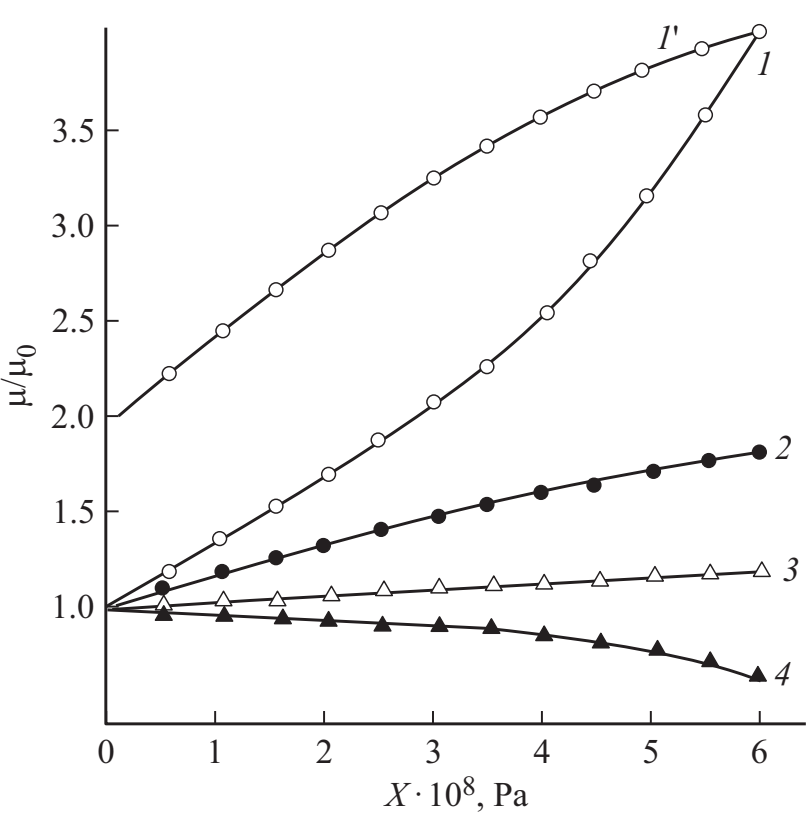

Рис. 3. Зависимости подвижности дырок в образцах $p-\mathrm{Si}\langle\mathrm{B}\rangle$ от одноосного сжатия при условиях $I\|X\|[111] \quad 1-$ TO $p$-Si $\langle\mathrm{B}, \mathrm{TD}\rangle, \rho=40 \Omega \cdot \mathrm{cm}, 2-$ исходный $p-\mathrm{Si}\langle\mathrm{B}\rangle$, $\rho=4 \Omega \cdot \mathrm{cm}, \quad 3-p-\mathrm{Si}\langle\mathrm{B}, \mathrm{Mn}\rangle, \quad \rho=7.7 \cdot 10^{2} \Omega \cdot \mathrm{cm}, \quad 4-$ $p-\mathrm{Si}\langle\mathrm{B}, \mathrm{Mn}\rangle, \rho=3.62 \cdot 10^{5} \Omega \cdot \mathrm{cm}$. 
нарушению локальной электронейтральности и, соответственно, появлению неоднородного крупномасштабного флуктационного потенциала. В узких, глубоких ямах и вершинах горбов потенциального рельефа искривленных зон полупроводника в зависимости от типа проводимости могут находится соответственно изолированные электроны и дырки, которые не участвуют в проводимости, а изменяют степень экранирования флуктационного потенциала [22,23]. В нашем случае с возрастанием одноосного сжатия, ямы и горбы потенциального рельефа смещаются соответственным образом, и концентрация дырок участвующих в проводимости увеличивается (рис. 2, кривая 4). В результате такого смещения изолированные электроны и дырки переходят в соответствующие уровни протекания, и взаимно компенсируются через центры рекомбинации. Уменьшение концентрации электронов в изолированных ямах приводит к ослаблению степени экранирования и, следовательно, к росту амплитуды флуктационного потенциала. Увеличение амплитуды флуктационного потенциала в свою очередь приводит к уменьшению подвижности дырок с ростом одноосного сжатия (рис. 3 , кривая 4).

\section{4. Выводы}

На основе полученных результатов можно сделать следующие выводы.

1. Проведенные исследования показали, что с помощью внешнего одноосного давления можно управлять концентрацией неоднородно распределенных примесных скоплений в термообработанных полупроводниках.

2. Тензочувствительность в компенсированных и СКобразцах $\mathrm{Si}\langle\mathrm{Mn}\rangle$ уменьшается в условиях сжатия $I\|X\|[111]$.

3. Эффект тензосопротивления в исходных образцах $p-\mathrm{Si}\langle\mathrm{B}\rangle$ обусловлен только изменением подвижности основных носителей тока, а в компенсированных и СКобразцах $p-\mathrm{Si}\langle\mathrm{Mn}\rangle$ изменением концентрации и подвижности основных носителей тока.

\section{Конфликт интересов}

Авторы заявляют, что у них нет конфликта интересов.

\section{Список литературы}

[1] M.K. Bakhadirkhanov, G.Kh. Mavlonov, S.B. Isamov, K.S. Ayupov, Kh.V. Iliev, O.E. Sattorov, S.A. Tachilin. Surf. Eng. Appl. Electrochem. 46, 3, 276 (2010).

[2] M.K. Bakhadirkhanov, K.S. Ayupov, G.Kh. Mavlonov, S.B. Isamov. Semicond. 44, 9, 1145 (2010).

[3] O.O. Mamatkarimov, O. Khimmatkulov, I.G. Tursunov. Semicond. 54, 5, 466 (2020).

[4] S. Zainabidinov, I.G. Tursunov, O. Khimmatkulov. Semicond. 52, 8, 1027 (2018).

[5] A.V. Fedosov, S.V. Luniov, S.A. Fedosov. Ukr. J. Phys. 55, 3, $322(2010)$.
[6] Ф.В. Федосов, С.В. Лунев, С.А. Федосов. Влияние одноосной упругой деформации на подвижность носителей тока в монокристаллах $n$-Si при наличии глубоких энергетических уровней. VIII Междунар. конф. „Взаимодействие излучений с твердыми телами“ (23-25 сентября 2009 г.) Минск, Беларусь.

[7] Г.П. Гайдар. ФТП 49, 9, 1164 (2015).

[8] В.В. Воронков, Г.И. Воронкова, А.В. Батунина, В.Н. Головина, Л.В. Арапкина, Н.Б. Тюрина, А.С. Гуляева, М.Г. Мильвидский. ФТТ 44, 4, 700 (2002).

[9] И.И. Новак, Г.А. Оганесян. Поверхность. Рентгеновские, синхротронные и нейтронные исследования 5, 82 (2007).

[10] Г.А. Оганесян, И.И. Новак. Поверхность. Рентгеновские, синхротронные и нейтронные исследования 12, 79 (2009).

[11] В.В. Емцев, Т.Б. Машовец, Г.А. Оганесян, К. Шмальц. ФТП 27, 9, 1545 (1993).

[12] 3.Н. Сальник. Неорган. материалы 31, 11, 1393 (1995).

[13] В.М. Бабич, Н.И. Блецкан, Е.Ф. Венгер. Кислород в монокристаллах кремния. Интерпрес ЛТД, Киев (1997). $240 \mathrm{c}$.

[14] M. Stavola, J.R. Patel, L.C. Kimerling, P.E. Freeland. Appl. Phys. Lett. 42, 73 (1983).

[15] P. Wagner, J. Hage, J.M. Trombetta, G.D. Watkins. Mater. Sci. Forum 83, 401 (1992).

[16] В.Л. Бонч-Бруевич, С.Г. Калашников. Физика полупроводников. Наука, М. (1990). 473 с.

[17] С.3. Зайнабидинов, О.О. Маматкаримов, И.Г. Турсунов, У. Туйчиев. ФТП 34, 6, 641 (2000).

[18] I.G. Tursunov. Ukr. J. Phys. 62, 12, 1041 (2017).

[19] М.К. Бахадырханов, С.Б. Исамов. Электрон. обработка материалов 46, 6, 8 (2011).

[20] S. Zainabidinov, O.O. Mamatkarimov, I.G. Tursunov, O. Khimmatkulov. Ukr. J. Phys. 62, 11, 957 (2017).

[21] I.G. Tursunov, O.O. Mamatkarimov, A.A. Okhunov. IIUM Eng. J. 19, 2, 164 (2018).

[22] Б.И. Шкловский, А.Л. Эфрос. Электронные свойства полупроводников. Наука, М. (1979). 367 с.

[23] А.Б. Давыдов, Б.А. Аронзон. ФТП 38, 6, 693 (2004).

Редактор К.В. Емцев 